\section{Optimiser la LAMal: Tout le monde il est bon, tout le monde il est gentil?}

\author{
Dr Yves Guisan, Vice-président FMH, Conseiller national
}

M. Otto Piller, Directeur de l'OFAS, fervent défenseur de la LAMal, essaie de nous faire avaler gentiment la pilule des contradictions de la loi, comme si vraiment il n'y avait qu'à introduire un budget global ambulatoire, une injuste clause du besoin malgré sa touchante compréhension de la situation de nos jeunes consœurs et confrères, et la magique concurrence économique pour faire baisser les prix à laquelle les patients s'empresseraient de contribuer.

Plutôt que de petits problèmes qui seraient si facilement résolus si les gentils partenaires le voulaient bien, il s'agit d'affronter les vrais et de cesser d'envoyer chaque automne les assurés au charbon. Les services de M. Piller ont eux-même contribué à l'élaboration des rapports IDAFISO I et II sur l'avenir des assurances sociales et les besoins de financement nouveaux à prendre en considération à l'avenir pour simplement maintenir l'acquis. En ce qui concerne l'assurance-maladie les projections font état de besoins supplémentaires compris entre $3 \%$ et 3,8\% en raison du vieillissement démographique et de l'allongement de l'espérance de vie. Ceci ne tient pas compte de l'introduction de nouvelles technologies d'un coût tout aussi élevé si ce n'est davantage. Avant de parler de transplantations, de robotisation, et autres techniques de pointe, que penser de la mise en place devenue routine des prothèses de hanche et de genoux, que penser des développements considérables de la chirurgie de l'épaule qui permet de faire face à des affections dégénératives que dans un passé encore tout proche il n'y avait qu'à supporter, que penser des formidables progrès dans le traitement du cancer dont nombre d'entre eux ne pouvaient aboutir qu'à une condamnation omineuse et sont maintenant guéris ou accessibles à des rémissions spectaculaires? Ne fût-ce que pour ces raisons incontournables les coûts de la santé ne peuvent qu'augmenter de manière très significative avec des primes d'assurance qui seront sans aucun doute d'un tiers plus élevées d'ici 2010 si l'on se réfère aux seuls calculs de l'administration fédérale. Dans ces conditions faire croire aux gentils assurés qu'elles devraient évoluer en fonction de l'indice des prix à la consommation, que l'on pourra toujours tout payer à tout le monde et qu'il n'y aura pas de place pour la médecine à deux vitesses est un miroir aux alouettes.

\section{Les contradictions de la LAMal}

Plutôt que de recourir à des mesures mesquines comme la levée de "l'obligation de contracter" pour les médecins de plus de 65 ans qui pratiquent dans la règle une médecine très économique à la satisfaction de leurs patients au gré de la «bouteille» que de nombreuses années d'expérience leur ont permis d'accumuler, il y a lieu de s'attaquer aux contradictions fondamentales de la LAMal.

\section{Transparence pour tous, pas seulement les médecins et les hôpitaux!}

Pour cela il est nécessaire avant tout d'établir une transparence complète à tous les niveaux et indispensable au rétablissement de la confiance, en particulier celui des caisses-maladie. Il n'y a pas de raison de les traiter différemment des hôpitaux dont l'organigramme, la grille des salaires, et les comptes sont connus dans leurs moindres détails. De son côté TarMed permettra de connaître exactement l'importance des prestations fournies en fonction de la morbidité.

\section{Redéfinir les règles de la concurrence}

La concurrence économique voulue par le législateur en méconnaissance des caractéristiques propres du marché de la santé est fondamentalement contradictoire avec un système d'assurance sociale solidaire et égalitaire. Les règles de l'économie sont mises en défaut par les multiples facteurs sociaux et éthiques à prendre en considération. A cela s'ajoutent des politiques cantonales diverses alors que bien des cantons n'atteignent même pas une taille macro-économique suffisante pour constituer un semblant de marché. Enfin jamais la concurrence économique n'a permis de faire baisser les coûts de la santé dans aucun pays du monde. Celle acharnée que se livrent les caissesmaladie malgré les divers garde-fous mis en place (fonds de compensation) n'a jusqu'à présent pas abouti à des résultats bien probants en dehors de surcoûts administratifs évidents. En l'occurrence la vraie concurrence ne repose que sur la qualité et rien d'autre. L'assurance sociale ne peut donc obéir qu'à une gestion régulatrice et normative. Mais avant de redéfinir les règles, encore faut-il distinguer clairement ce qui relève de l'assurance sociale et solidaire par opposition aux prestations de confort individuel.

\section{Redéfinir les compétences fédérales/cantonales}

La réponse à ces questions fondamentales sur le plan des principes et sur le plan opérationnel ne demande pas seulement la transparence, mais aussi une répartition des compétences dépourvue de toute équivoque. Dans l'actuelle LAMal, «le Conseil fédéral règle tous les détails», mais pour finir aucun parce qu'il 
butte presque systématiquement sur les compétences ou l'inertie des cantons. Et pour finir ce sont les tribunaux qui font la politique de la santé. Il est temps de mettre de l'ordre dans cet imbroglio afin d'établir clairement les procédures et de savoir qui est le chef et qui fait quoi. C'est la condition sine qua non d'une politique de santé efficace.

\section{Halte au rafistolage!}

On est malheureusement loin d'en prendre le chemin. $\mathrm{Au}$ contraire, de peur de se brûler les doigts en touchant à l'essentiel, on recourt à des rafistolages au coup par coup. Comment imaginer de réviser le financement des hôpitaux en proposant une solution qui met à la charge des cantons 600 millions supplémentaires et un supplément de prime de près de $10 \%$ à celle des assurés? On croit véritablement rêver! Le remède devient pire que le mal! Quant à une collaboration des organisations de patients pour résoudre ces problèmes, le corps médical ne peut que s'en réjouir. Mais il est permis de douter qu'elle permette de régler en un tour de main le dilemme entre le traitement optimal et sans considération de prix que souhaite le malade et les primes les plus avantageuses possibles que souhaitent les assurés.

Optimiser la LAMal, oui, mais avec une gestion transparente et une stratégie cohérente digne d'une véritable politique de santé, pas celle de «La guerre des boutons"*!

\footnotetext{
* La guerre de boutons. Film d'Yves Robert (1961) où deux bandes rivales d'enfants s'arrachent les boutons de manière à ce que le vaincu perde ses culottes!
} 